\title{
Color Image Segmentation Using Improved Method of Normal Cut
}

\author{
Adnan Ahmad ${ }^{1}$, Guo Ling ${ }^{1, *}$ and Hassan Hayat ${ }^{2}$ \\ ${ }^{1}$ School of Automation, Nanjing University of Science and Technology \\ ${ }^{3}$ School of Computer Science and Engineering, Nanjing University of Science and Technology
}

\begin{abstract}
Image segmentation is a key problem in several fields of science and technology, for E.g. medicine, robotics, and industrial development. The importance of perceptual grouping and organization in vision possess numerous significant factors, such as similarity, proximity, and good continuation, which lead towards the visual grouping in present. However, even to this day, many of the computational issues of perceptual grouping have remained unresolved. This article presents image segmentation as a graph partitioning problem and proposed modified method of normalized cuts, for segmenting the graph. The normalized cut criterion measures both the total dissimilarity between the different groups as well as the total similarity within the groups. The proposed algorithm deals with the color images which give more clear understanding of an image as compared to the black and white images. The experimental results show that the proposed algorithm gives better, clear and more visible understanding of UAV images.
\end{abstract}

Keywords-grouping; image segmentation; graph partitioning; normalized cuts (ncut); graph theory

\section{INTRODUCTION}

Segmentation is used as a building block for problem solving and a critical phase in machine vision applications. The objective of the segmentation process is to cluster the points with similar characteristics into isolated homogenous regions. These isolated regions should be meaningful [1], [2], [3], [4]. The segmentation process could be helpful for analyzing the scene in various aspects such as locating and recognizing objects, classification, and feature extraction. It is relatively challenging to extract the knowledge (the high level information) directly from the low-level pixel collection. This idea can be utilized in further repartitioning or grouping [5], [6] [7], [8].The key point is that image partitioning is to be done from the big picture downward, rather like a painter first marking out the major areas and then filling in the details. Graph-based segmentation takes into account global image properties as well as local spatial relationships, and results in a region map that is ready for further processing, e. g., region merging or labeling. The proposed segmentation considers the points in a term of graph.

\section{RELEATED WORKS}

Reference [9] proposed a graph cut technique through we can easily and efficiently solve a wide variety of low-level computer vision problems, such as image smoothing and many other computer vision problems. The stereo correspondence problem can be solved in terms of energy minimization. We can solve the binary problems exactly by using this approach; here in this problem where pixels can be labeled with more than two different labels (such as stereo correspondence of a grayscale image) cannot be solved exactly, but the optimum solution of the problem can be achieved. Here, in the graph cut technique the image in the form of graphs is presented (i.e. containing nodes and vertices like graph), where each pixel acts as anode and the distance between those nodes presents the edges. Distance between the nodes is calculated by using the attributes of the Ohta's color model.

In graph theory, a cut is a partition of the nodes that divides the graph into two disjoint subsets. The set of cuts is the set of edges, whose ending points are different subsets of the divided regions. If edges are in its cut-set then they are said to be crossing the cut. In an un-weighted undirected graph, it can be described that the weight or size of a cut is the number of edges that are crossing the cut in an image and in the case of a weighted graph; it is defined as the sum of the weights of all the edges crossing the cut.

$\mathrm{Wu}$ and Leahy [9] proposed a clustering method based on this minimum cut criterion. In the minimum cut technique it is required to segment each and every pixel of an image. That means in this technique it is needed to cut each pixel in an image even if those pixels are similar with respect to color, intensity or texture. So in this minimum cut technique it was difficult to get a better segmented image when compared to the other techniques. In particular, they want to partition a graph into k-sub graphs such that the maximum cut across the subgroups is minimized.. This problem can be solved by recursively finding the minimum cuts which bisect the existing segments. As shown in Wu and Leahy's work, this globally optimal criterion can be used to produce good segmentation on some of the images. The minimum cut criteria favors cutting the small sets of isolated nodes in the graph.

Assuming that edge weights are inversely proportional to the distance between the two nodes, we observed the cut that partitions out node $\mathrm{n} 1$ or $\mathrm{n} 2$ possesses a very small value. In fact, any cut that partition out individual nodes on the right half will have smaller cut value than the cut that partitions the nodes into the left and right halves. In minimized cut technique we couldn't avoid the unnatural bias for cutting small set of points. To avoid this unnatural bias for partitioning out small sets of points, they proposed a new measure of disassociation [9]. 


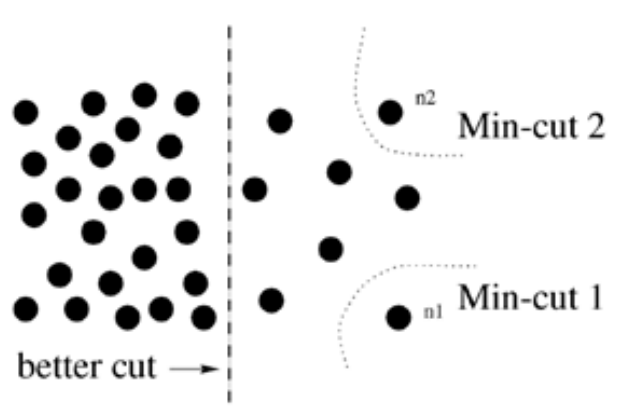

FIGURE I. A CASE WHERE MINIMUM GIVES A BAD PARTITION[10]

The normalized cut method was proposed by J. Malik and J. Shi [10]. In their view, the image segmentation problem can be associated as a graph theory problem. Graph theory is an interesting math topic which models math problems into edges and vertexes.. Each edge can contain a value (weight), which can be used as flow. This kind of graph is called "weighted graph".
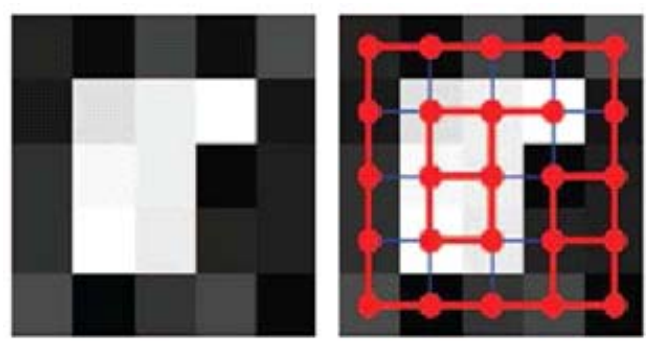

\section{FIGURE II RIGHT SIDE (ORIGIONAL IMAGE) LEFT SIDE (GRAPH} IMAGE REPRESENTATION)

In Fig. 2 two images are represented, where first image is the original image and the second image is represented in the form of graph. In the above image thick edges represent the strong similarities between two pixels and thin edges represent the week similarities between the two pixels. In the graph cut technique the image is cut from the places where weak similarities are available in between the two pixels.

This is a connected graph because each pixel can go through the edges to reach all other pixels. The term "cut" refers to eliminating a set of edges in an image to make the graph "unconnected", and the value of the cut is the total weights on this set of edges. For example, if we eliminate all the thick edges in Fig. 2, then the nodes with white color will be "unconnected" to the nodes with dark color, and now it can be said that the graph has been separated into two connected parts. So, from the graph theory the image segmentation problem is modeled as graph cut problem. The weights on edges have the similarity between pixels, so if we want to divide two pixels into two different regions, their similarity is expected to be very small.

The purpose of this research work is to introduce a modified method of normal cuts, which will not only allow the gray scale image but will also represent a comprehensive explanation in the form of the color image with greater accuracy.

\section{IMPROVED METHOD OF NORMAL CUTS}

The basics of modified method of normal cuts algorithm are similar to the previously described algorithms [10]. However, the peculiarities are associated with the processing of color images. The image is presented as a weighted graph (Fig. 3), with vertices at the points. The weight of a graph edge reflects similar measures (distance between points calculated by some metric).
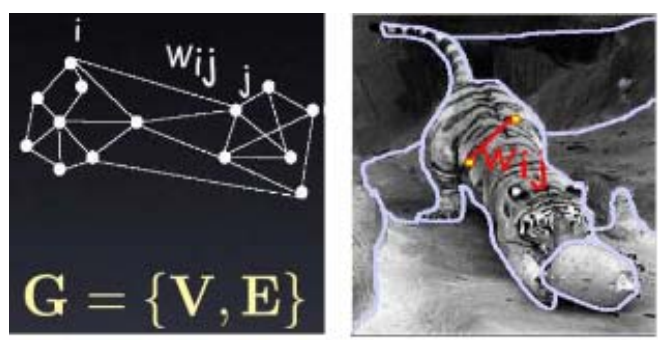

\section{FIGURE III. AN EXMPLE OF MODELING A GRAPH}

In method [10], every element of the collection, which is considered to be divided into clusters, must be correlates with the vertex of the graph. Furthermore, all pairs of vertices are constructed by edges and the weight ratio, which represent a degree of similarity elements. Then the edges of the graph are cut by a manner, which combines all the related elements in one set. In an ideal case, the weighting coefficients of the edges inside the components should be large as compared to the coefficients of the connecting edges.

Each component presents a cluster, and weight coefficients are usually called the measures of similarities between them. There are different approaches to measure the similarity such as the intensity and color. The core of the method lies in the fact that cut the two related components so that in each group, the cost of cutting is small in comparison with the general similarity. This approach can be formalized by dividing weighted graph $V$ into two components $A$ and $B$.

\section{A. Formula Explanation}

$$
\begin{aligned}
& \operatorname{Ncut}(A, B)=\frac{\operatorname{cut}(A, B)}{\operatorname{assoc}(A, V)}+\frac{\operatorname{cut}(A, B)}{\operatorname{assoc}(B, V)} \\
& =2-\left(\frac{\operatorname{assoc}(A, A)}{\operatorname{assoc}(A, V)}+\frac{\operatorname{assoc}(B, B)}{\operatorname{assoc}(B, V)}\right)
\end{aligned}
$$

In Eq. (1), $\operatorname{cut}(A, B)$ is the sum of the weighted coefficients of all edges of the graph $V$, one end which lies in $A$, and the other in $B$.

$$
\operatorname{cut}(A, B)=\sum_{u \in A, v \in B} w(u, v)
$$

$\operatorname{assoc}(A, V)$ is the sum of the weighting coefficients. 


$$
\operatorname{assoc}(A, V)=\sum_{u \in A, t \in B} w(u, t)
$$

This parameter is small; if the cutting of two components is obtained that have small edges with small weight coefficients. Minimization of the Eq. (1) reduces to the following:

$$
\min _{x} \operatorname{Ncut}(x)=\left(\frac{\min _{y}\left(y^{T}\right)(D-W) y}{y^{T} D_{y}}\right)
$$

Under the conditions $y_{t} \in\{1,-b\}, 0<b \leq 1$ and $y^{T} D 1=0$ The $\mathrm{x}$ is the indicator vector. $x_{i}=1$ If the vertex is in $A$ and -1 Otherwise; $W$ is the matrix of weights. $W(i, j)=w(i, j)$ And $D$ is the diagonal matrix.

$$
b=\frac{\sum_{i}>0}{\sum_{i} d_{i}}
$$

If $y \in \mathfrak{R}$ (Rayleigh Quotient) then the Eq. (2) reduces to the eigenvalue problem:

$$
(D-W)_{y}=\lambda D_{y}
$$

According to Eq. (1-3) the segmentation algorithm consists of following steps:

1. In the image, you must specify a graph and weights matrix.

$$
W(i, j)=w(i, j)
$$

2. There are vectors with smallest eigenvalues. (It solves Eq. (2), (3)).

3. For a vector with the second smallest eigenvalue, the Value is calculated from the phase into two parts.

4. If necessary obtained areas recursively break into sub-regions. Each pair of pixels is checked for the presence of a boundary on the line connecting them. If there is a boundary, its intensity is equal to the image gradient and is used for the calculation of the weighting coefficient of the boundary. $w_{\text {edge }}=\frac{-f^{2}}{e^{2 \sigma^{2}}}$

(where $\sigma$ is the $10 \%$ of the boundary intensities)

Weighting factor is:

$$
w=w_{\text {edge }} \cdot w_{\text {colour }}
$$

Since the essential differences in the shades represent the different objects, so the proximity of shades criteria is used. Here it is noted that the shade persists even in the parts of an image. The pixels are calculated by the conversion of the source image in the color space HSV (Hue, Saturation, Value). In the case of a significant differences in shades (more than 30 degrees), the weighting factor is multiplied by the factor 0.01 .

\section{RESULTS}

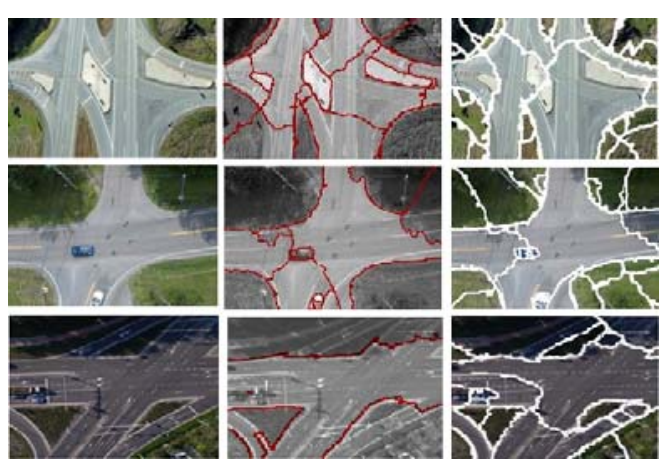

FIGURE IV LEFT (ORIGIONAL IMAGE) MIDDLE (ORIGIONAL NORMAL CUT [10]) RIGHT (IMPROVED NORMAL CUT)

Fig. 4 clearly shows the results of the two methods, one is proposed by [10] and second is the normalized image of modified cut method. It is clear from Fig. that method proposed by J. Malik and J. Shi [10] only converts the image in a small number of segments(gray scale mode), which inevitably leads towards the loss of accuracy, whereas the modified method deals efficiently with color images and has segmented the image into small regions. This algorithm gives the ease to segment the road from UAV images.

\section{CONCLUSION}

The research work presents a modified algorithm of normal sections, which improves the characteristics of the original method. Comparative experiments show that the modified method of normal cuts gives the output much accurate than the standard method which improves the accuracy of segmentation. The standard normal cut algorithm works only on black and white images rather than the color images. The modified algorithm deals with the color images which gives more understanding of an UAV (Unmanned Autonomous Vehicle) image.

\section{ACKNOWLEDGMENT}

This work was supported in part by the National Natural Science Foundation of China, in part by the Natural Science Foundation of Jiangsu province, Doctoral Fund through the Ministry of Education, China, in part by the Program of Introducing Talents of Discipline to Universities.

\section{REFERENCES}

[1] A.K. Jain and R.C. Dubes, Algorithms for Clustering Data. Prentice Hall, 1988.

[2] Borel, A. Tremeau and N.A, Region growing and Merging Algorithm to color segmentation. [Pattern Recognition], Vol. no.30 (7), pp. 11911203, 1997.

[3] Y.Kanai, Image Segmentation Using Intensity and Color Information. [SPIE -Visual Communications and Image Processing], vol. 3309, pp. 709-720, San Jose, CA, 1998. 
[4] Cramariuc, B., Gabbouj, M. Astola,J. Clustering Based Region Growing Algorithm for Color Image Segmentation.[13th Int. Conf. on Digital signal Processing], vol. 2, pp.857-860, 1997.

[5] Deng, Y., Manjunath, B. S. Shin, H, Color Image Segmentation, IEEE Computer Society Conference on Computer Vision and Pattern Recognition CVPR, vol.2,pp.446-451, 1999.

[6] Jain, R., Kasturi, R. Schunck, B.G. Machine Vision, McGraw-Hill, 1995

[7] Bow, S.-T, Pattern Recognition and Image Processing ,Marcel Dekker, Inc.,NewYork,NY,1992.

[8] [8] R.M., Haralick L.G.,Shapiro, Image Segmentation Techniques, Computer Vision, Graphics, and Image Processing T.1, Vol. 29, pp. 100$132,1985$.

[9] [9] Z. Wu and R. Leahy, An Optimal Graph Theoretic Approach to Data Clustering: Theory and Its Application to Image Segmentation, IEEE Trans. Pattern Analysis and Machine Intelligence, vol. 15,no. 11, pp. 1,101-1,113, Nov. 1993.

[10] [10] Shi J., Malik J, Normalized Cuts and Image Segmentation IEEE Transactions on Pattern Analysis and Machine Intelligence, vol. 22, No.8, pp. 888-905, 2000 